\title{
Comportamiento viscoelástico de la madera de Prosopis sp.
}

\author{
Javier Ramón Sotomayor Castellanos ${ }^{1}$ \\ y José María Villaseñor Aguilar ${ }^{1}$
}

\begin{abstract}
RESUMEN
Los elementos estructurales de madera sometidos a cargas permanentes sufren deformaciones, lo que denota un carácter viscoso del material. Con el objeto de asegurar la fiabilidad de la estructura es necesario anticipar un comportamiento conjunto que resulta de la respuesta elástica y del comportamiento diferido de la madera. El objetivo de la investigación es demostrar el carácter viscoelástico de la madera, manifestado por la diferencia entre los valores del Módulo de Elasticidad estático y dinámico de una muestra de probetas normalizadas de Prosopis $\mathrm{sp}$. Se realizaron pruebas no destructivas de flexión transversal con dos variantes: vibración en apoyos simples (ensayo dinámico) y flexión en tres puntos (ensayo estático). Los resultados demostraron que el Módulo de Elasticidad estático (MOE) es un buen predictor de Módulo de Elasticidad dinámico (Ed) y viceversa. Además, el alto coeficiente de correlación encontrado entre estos parámetros, postula que si aumenta el valor del MOE de la madera de Prosopis sp., su valor de Ed se incrementa proporcionalmente. Los ensayos dinámicos practicados resultaron ser confiables, fueron más rápidos y sus parámetros medidos fueron más repetitivos en comparación con los ensayos estáticos. La investigación fue un estudio de caso y una consecuencia de estos resultados es que la aplicación de la metodología es posible para otras especies y dimensiones de probetas.
\end{abstract}

PALABRAS CLAVE:

Módulo de Elasticidad estático y dinámico, Prosopis, viscoelasticidad.

\begin{abstract}
Wooden structural elements that support permanent loads bear mechanical strains. This indicates the viscoelastic nature of this material. In order to guarantee the structure's reliability it is necessary to predict the elastic and viscous response of wood in service. The objective of this research is to show the viscoelastic nature of wood, which in turn is manifested by the difference between the Static and Dynamic Modulus of Elasticity values of a standard sample of Prosopis sp. wood. Non-destructive tests were carried out by following two procedures: transverse flexural vibration and static bending. Results showed that the Static Modulus of Elasticity (MOE) is a good predictor of the Dynamic Modulus of Elasticity (Ed) and viceversa. In addition, the high coefficient of correlation found between these parameters denotes that if the value of MOE of Prosopis sp. wood increases, the value of its Ed increases proportionally. The dynamic tests were reliable, fast and the parameters measured were more repetitive in contrast with the Static tests. This research was a case study. As a consequence of this study, the methodology for studying other wood species with different specimen's geometry is possible.
\end{abstract}

KEYWORDS:

Static and dynamic Modulus of Elasticity, Prosopis, viscoelasticity. 


\section{INTRODUCCIÓN}

La madera es un material ampliamente usado en construcción, que se comporta mecánicamente como un sólido elástico y que obedece las leyes de la resistencia de materiales. Sin embargo, al paso del tiempo los elementos estructurales de madera sometidos a cargas permanentes sufren deformaciones, lo que denota un carácter viscoso en la madera. Este comportamiento depende de la intensidad y duración de la carga y de la rigidez del miembro estructural, entre otros factores. Con el objeto de asegurar la fiabilidad de la estructura, es necesario anticipar un comportamiento conjunto que resulta de la respuesta elástica y del comportamiento diferido de la madera.

El desarrollo de productos fabricados con madera requiere, además, información normalizada y confiable de las propiedades de este material. Una de las características mecánicas más necesarias en Ingeniería de la Madera es el Módulo de Elasticidad, el cual encuentra su utilidad como parámetro de diseño, cálculo e indicador de calidad.

De acuerdo con Ross y Pellerin (1994), usualmente el Módulo de Elasticidad dinámico $(\mathrm{Ed})$ de la madera es determinado empleando métodos de evaluación de carácter no destructivo, por ejemplo: ondas de esfuerzo y ultrasonido, entre otros. Para este estudio se utilizaron vibraciones mecánicas. Con el objeto de caracterizar a la madera, el Módulo de Elasticidad dinámico se utiliza en la verificación de los métodos de laboratorio referentes a la calidad de resultados obtenidos en ensayos mecánicos, de acuerdo con el Manual de Construcción con Madera del Instituto Americano de Construcción con Madera (American Institute of Timber Construction, 1994).
La caracterización del comportamiento mecánico de la madera en condiciones dinámicas, y los métodos de laboratorio recientemente desarrollados son de carácter no destructivo y han utilizado la hipótesis fundamental en mecánica de la madera propuesta por Jayne en 1959: la madera y los productos fabricados con ella pueden almacenar y disipar energía; por ejemplo, la propiedad de la madera de almacenar energía es manifestada por la velocidad a la cual una onda mecánica viaja a través de ella. En contraste, la capacidad de la madera para atenuar una onda de vibración denota su capacidad para disipar energía. Jayne propuso así la hipótesis de que estas propiedades de la madera para almacenar y disipar energía están controladas por los mismos mecanismos que determinan su comportamiento mecánico en condiciones estáticas. Es decir, la estructura molecular y anatómica del material es la base del comportamiento mecánico de la madera. Como consecuencia, es posible relacionar estadísticamente estas propiedades utilizando métodos de análisis numéricos como las correlaciones estadísticas. Esta proposición ha sido verificada experimentalmente por los trabajos de Jayne (1959); Pellerin (1965); Kaiserlik y Pellerin (1977); Ross y Pellerin (1988); Ross et al. (1977); y más recientemente por Sandoz (2000 y 2002).

Por otra parte, a partir de la teoría propuesta por Timoshenko et al. (1994), sobre la resistencia de materiales aplicada al estudio de vigas en ingeniería, Hearmon (1966) estudió el comportamiento anisotrópico de la madera y la relación de esbeltez de las probetas en un ensayo de flexión en vibración transversal. A partir de sus resultados empíricos, el autor formuló el procedimiento experimental, utilizado posteriormente por diferentes autores en estudios sobre el comportamiento elástico de la madera. Sus valores de laboratorio del Módulo de 
Elasticidad para Fraxinus excelsior, a un contenido de humedad del $12 \%$, determinado en un ensayo de flexión en vibración transversal fue de $14 \mathrm{GPa}$, y para el Módulo de Elasticidad en flexión estática fue de $10 \mathrm{GPa}$, es decir, una diferencia de $40 \%$.

Con el propósito de confirmar la utilidad de la teoría de análisis de vigas, propuesta por Goens (1931) y Timoshenko et al. (1994), Perstorper (1992) comparó el Módulo de Elasticidad en vigas de dimensiones estructurales de Picea excelsa, aplicando ensayos de vibración transversal y de flexión estática. Sus resultados confirman que hay un $8 \%$ de diferencia entre el Módulo de Elasticidad dinámico y el Módulo de Elasticidad estático, siendo mayor el dinámico.

Los resultados encontrados por Perstorper (1992) en Picea excelsa, a un contenido de humedad del $12 \%$ para el Módulo de Elasticidad dinámico fue de 9 GPa y para el Módulo de Elasticidad estático (MOE) fue de $8 \mathrm{GPa}$, es decir, una diferencia del 12,5\%. Además, el autor encontró regresiones entre los valores dinámicos y estáticos con coeficientes de correlación en promedio de 0,94 , confirmando de esta manera la relación entre los dos parámetros calculados con métodos donde solo varía la velocidad de la aplicación de la carga.

Respecto a la utilización de tecnologías de carácter no destructivo y de su aplicación en el estudio de la madera, Görlacher (1984) realizó pruebas de flexión en vibración transversal sobre probetas normalizadas, utilizando la tecnología GrindoSonic ${ }^{\circledR}$ y la metodología desarrollada anteriormente por Kollmann y Krech (1960) y Hearmon (1966). El autor comprobó la utilidad de este método experimental para la determinación del Módulo de Elasticidad en flexión transversal por vibración en la madera. Los datos del Módulo de Elasticidad dinámico de $15 \mathrm{GPa}$ fueron superiores en 7\% comparados al Módulo de Elasticidad estático de $14 \mathrm{GPa}$.

Haines et al. (1996) determinaron el Módulo de Elasticidad en flexión para un contenido de humedad del $12 \%$, en vibración transversal para la madera de Picea excelsa que resultó en $11 \mathrm{GPa}$ y en Abies amabilis el cual fue de $13 \mathrm{GPa}$. Sus resultados demostraron que el Módulo de Elasticidad dinámico calculado para madera estructural fue $6 \%$ superior al Módulo de Elasticidad estático y para probetas de pequeñas dimensiones la diferencia respectiva fue de $3 \%$. Los autores hacen notar que las propiedades viscoelásticas de la madera influyen en la diferencia de valores entre los Módulos de Elasticidad dinámicos y estáticos.

Por su parte, Ilic (2001) estudió la relación entre los valores dinámicos y estáticos provenientes de ensayos de flexión transversal en probetas de pequeñas dimensiones, a un contenido de humedad del $12 \%$ de Eucalyptus delegatensis, y encontró que el Módulo de Elasticidad dinámico de 16 GPa es mayor que el Módulo de Elasticidad estático de 14 GPa en $14 \%$.

De la revisión de autores sobre el tema en estudio se puede sintetizar que los métodos de flexión en vibración transversal y flexión estática se han utilizado con éxito para determinar el Módulo de Elasticidad de la madera, y que el valor dinámico es generalmente mayor al estático.

De acuerdo con Ashby (1999), el Módulo de Elasticidad es una característica intrínseca del material y su valor no debe variar según el método de ensayo utilizado para su determinación. Sin embargo, en experimentos para la determinación del Módulo de Elasticidad, la madera denota diferentes valores si la 
velocidad de carga varía. Esta diferencia en los resultados, para una propiedad intensiva como el Módulo de Elasticidad de la madera en flexión transversal, puede ser explicada por su carácter viscoelástico, criterio confirmado por Panshin y De Zeeuw (1964), Kollmann y Côté (1968), Guitard (1987) y Bodig y Jayne (1993), entre otros autores, y documentada en el Manual de la madera como material de Ingeniería del Laboratorio de Productos Forestales de los Estados Unidos de América (Forest Products Laboratory, 1999).

\section{OBJETIVO}

Demostrar el carácter viscoelástico de la madera manifestado por la diferencia entre los valores del Módulo de Elasticidad de una muestra de probetas normalizadas de Prosopis sp., medidos en un ensayo de flexión transversal, en condiciones de carga dinámica y estática.
La investigación se fundamenta en la Teoría de la Resistencia de Materiales y en la Teoría de Vibraciones. Además, la proposición se concreta a su comportamiento en solicitaciones de flexión estática en tres puntos y dinámica en vibración transversal sobre apoyos simples, en condiciones de invariabilidad térmica.

\section{METODOLOGÍA}

El material experimental se obtuvo de una troza de un árbol de Prosopis sp. (mezquite) recolectado en la región de Dolores Hidalgo, Guanajuato. Las dimensiones de la troza, esquematizada en la Figura 1, fueron $200 \mathrm{~mm}$ de diámetro por $400 \mathrm{~mm}$ de largo. Las pruebas se efectuaron en la Universidad Michoacana de San Nicolás de Hidalgo, durante el segundo semestre del 2004.

A partir de cada uno de los seis sectores en que se dividió la troza, se

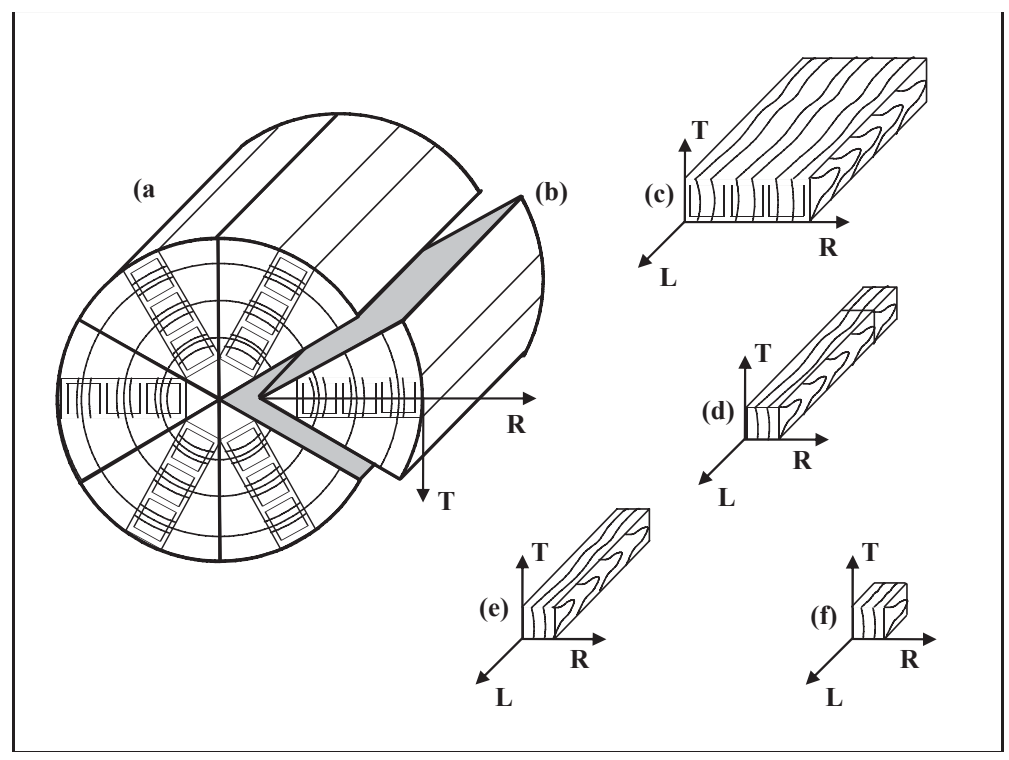

Figura 1. Selección de probetas. a) Troza; b) Sector; c) Segmento; d) Listón; e) Probeta ensayo de flexión; f) Probeta densidad y contenido de humedad. 
recortaron segmentos en forma de paralelepípedo, adaptando la norma Iso 31291975 (International Organization for Standardization, 1975a) con dimensiones de $22 \mathrm{~mm} \times 72 \mathrm{~mm} \times 400 \mathrm{~mm}$, como se muestra en la Figura 1. Con el objeto de estabilizar el contenido de humedad en la madera, los segmentos se mantuvieron en una cámara de acondicionamiento en condiciones constantes, a temperatura de $20^{\circ} \mathrm{C}$ y humedad relativa de $65 \%$, durante un periodo de 65 días, hasta alcanzar un contenido de humedad (C.H.) final de $12 \%$.

Al término del periodo de acondicionamiento, de cada segmento orientado en la dirección radial, se prepararon tres listones, con dimensiones de
$22 \mathrm{~mm} \times 22 \mathrm{~mm} \times 400 \mathrm{~mm}$, a partir de los cuales se elaboraron las probetas normalizadas para los ensayos de flexión dinámicos y estáticos no destructivos con dimensiones de $20 \mathrm{~mm}$ x $20 \mathrm{~mm} \times 320 \mathrm{~mm}$ de acuerdo a la norma Iso 3349-1975 (International Organization for Standardization, 1975b), como se ve en la Figura 1.

A cada una de las 18 probetas que constituyeron la muestra de estudio, se le midieron sus dimensiones: largo (L), base (b) y altura $(h)$, y masa $(m)$, y se calculó: volumen $(\mathrm{V})$, densidad (ñ), momento de inercia de la sección transversal (I) y contenido de humedad (C.H.) al momento del ensayo (ver Figura 2).

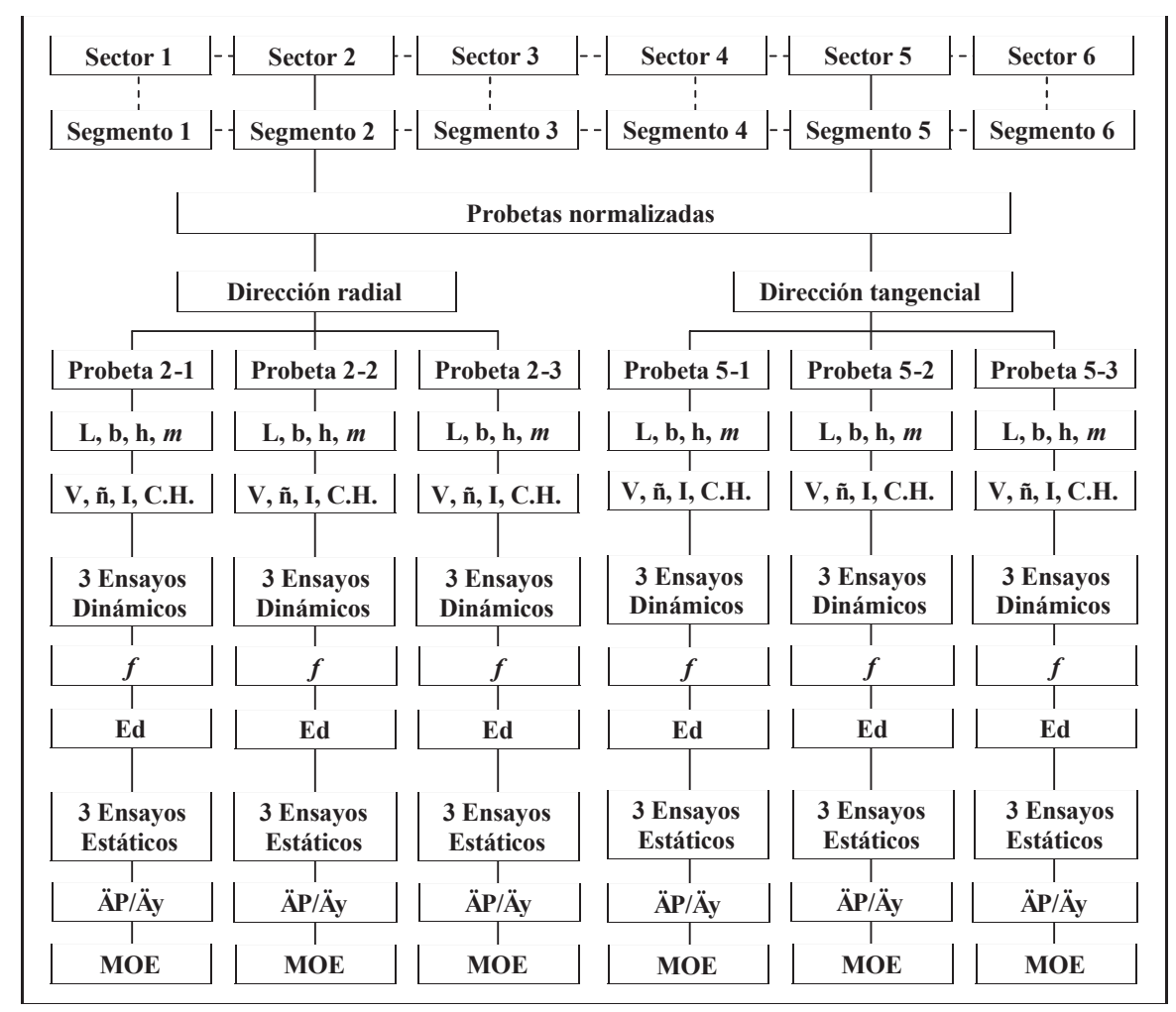

Figura 2. Estrategia experimental para ensayos no destructivos. 
El total de 18 probetas se dividió en dos grupos de nueve especímenes cada uno: el grupo radial y el grupo tangencial, con el objeto de girar su posición 90 grados en el plano R-T (ver Figura 2) en los ensayos de flexión, tal como se esquematiza en la figuras 3 y 4 .

A todas las probetas, se les realizaron pruebas no destructivas de flexión transversal con dos variantes: vibración en apoyos simples (Ensayo dinámico) y flexión tres puntos (Ensayo estático) efectuando tres ensayos dinámicos y estáticos, respectivamente, en cada probeta. Esta estrategia experimental se ilustra en la Figura 2.

\section{Ensayos dinámicos no destructivos}

Los ensayos dinámicos no destructivos consistieron en el estudio de la probeta modelada como una viga continua, de geometría uniforme y estructuralmente homogénea, sometida a vibración transversal. La rutina de laboratorio siguió los lineamientos del manual de operación del equipo GrindoSonic ${ }^{\circledR}$ (Lemmens, s/f). La Figura 3 muestra el montaje de laboratorio y el sensor con el cual se registró la vibración de la viga y se midió la frecuencia natural del sistema.

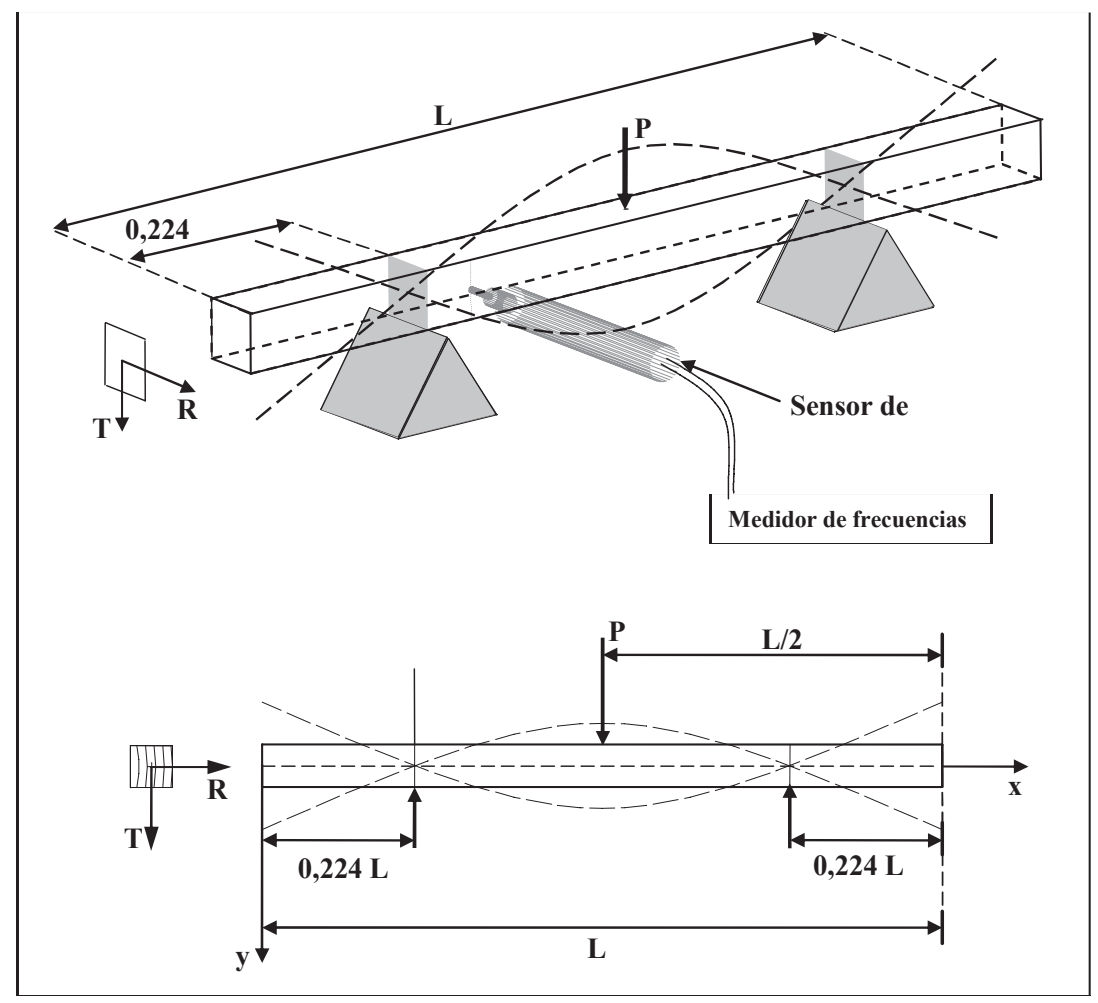

Figura 3. Esquema ensayo dinámico y diagrama de la probeta. 


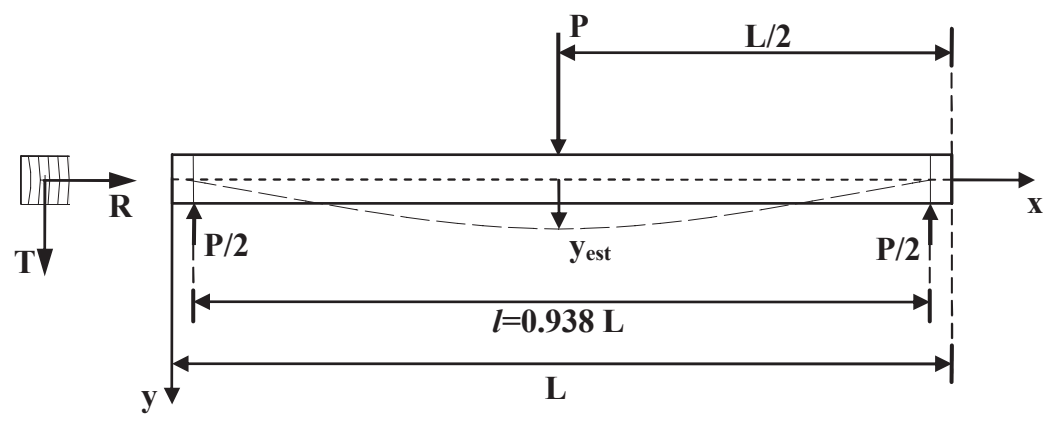

Figura 4. Diagrama de la probeta en el ensayo estático en tres puntos.

El impulso elástico inicial fue aplicado en el centro geométrico de la probeta, en la dirección transversal a la misma, apoyada sobre dos soportes rígidos de tipo simple, a una distancia nodal de 0,224 L. El ensayo dinámico en cada probeta fue repetido tres veces y el promedio de valores fue considerado para su análisis posterior.

La fórmula utilizada para la determinación del Módulo de Elasticidad dinámico fue presentada por Goens (1931), y viene dada por:

$$
E d=\frac{4 \pi^{2} L^{4} f^{2} \rho}{m^{4} r^{2}}\left(1+\frac{r^{2}}{L^{2}} K\right)
$$

donde:

$E d=$ Módulo de Elasticidad dinámico de la madera $(\mathrm{Pa})$.

$L=$ Longitud de la probeta $(\mathrm{m})$.

$f=$ Frecuencia natural de la probeta $(\mathrm{Hz})$.

$\rho=$ Densidad de la probeta $\left(\mathrm{kg} / \mathrm{m}^{3}\right)$.

$m, K=$ Constantes.

$r=$ Radio de giro de la sección transversal de la probeta $\left(\mathrm{m}^{2}\right)$.

\section{Ensayos estáticos no destructivos}

Las pruebas estáticas no destructivas consistieron en el estudio de la probeta sometida a una carga estática aplicada transversalmente en el centro de su portada de ensayo. Tal como se muestra en la Figura 4, que presenta el diagrama de la viga sometida a una solicitación de flexión tres puntos.

El proceso de carga-deformación en los ensayos estáticos no destructivos fue restringido a un intervalo de carga que representó una fracción entre 18\% y 35\% del dominio elástico. Esta estrategia aseguró el carácter de evaluación no destructivo en los ensayos estáticos. El ensayo estático en cada probeta fue repetido tres veces y el promedio de valores fue considerado para su análisis posterior.

La fórmula simplificada que se aplicó para el cálculo del Módulo de Elasticidad estático fue (Bodig y Jayne, 1993):

$$
M O E=\frac{\Delta P}{\Delta y} \frac{l^{3}}{48 I}
$$


donde:

MOE = Módulo de Elasticidad estático de la madera $(\mathrm{Pa})$.

$\triangle \mathrm{P}=$ Intervalo de carga, ensayos no destructivos $(\mathrm{N})$.

$\Delta y=$ Intervalo de deformación, ensayos no destructivos $(m)$.

$I=$ Portada del ensayo $(\mathrm{m})$.

$\mathrm{I}$ = Momento de inercia de la sección transversal de la probeta $\left(\mathrm{m}^{4}\right)$.

\section{RESULTADOS}

La tabla 1 presenta las estadísticas Media muestral, Error estándar, Asimetría, Curtosis y Tamaño de la muestra del Módulo de Elasticidad dinámico (Ed) y del Módulo de Elasticidad estático (MOE), para probetas normalizadas.

La Figura 5 muestra el gráfico de los valores del Módulo de Elasticidad dinámico (Ed) y del Módulo de Elasticidad estático (MOE) para cada una de las 18 probetas estudiadas.

La Figura 6 muestra el diagrama de dispersión entre los Módulos de Elasticidad estático y dinámico y su línea de regresión.

\section{DISCUSIÓN Y CONCLUSIONES}

El análisis del comportamiento de las probetas, acorde con su rotación en el plano R-T, demostró que la rotación de las probetas en relación a la dirección de la carga, no influye de manera importante en la magnitud de los valores de Ed y MOE para este estudio. El no rechazo de la hipótesis nula $\left(\mathrm{H}_{0}: \mathrm{i}_{1}=\mathrm{i}_{2}\right)$, para las muestras Ed y MOE, radial y tangencial, y las correlaciones derivadas entre los valores de los módulos permiten, por una parte, agrupar para análisis subsecuentes los resultados de los dos grupos de nueve probetas (según su orientación radial y tangencial), en un sólo conjunto de dieciocho especímenes. Además, los valores de asimetría y curtosis que se presentan en la tabla 1 permiten considerar a los estadísticos de Ed y MOE como provenientes de una distribución normal para un nivel de confiabilidad del $95 \%$. A partir de estos resultados, en la tabla 1 se presentan los estadísticos para las 18 probetas agrupadas indistintamente de su orientación en el ensayo de flexión.

El valor calculado de la media muestral del Módulo de Elasticidad dinámico (Ed) para madera de Prosopis sp., que se

Tabla 1. Estadísticas del Módulo de Elasticidad dinámico (Ed) y Módulo de Elasticidad estático (MOE).

\begin{tabular}{lcc}
\multicolumn{1}{c}{ Estadígrafos } & $\begin{array}{c}\mathrm{Ed} \\
(\mathrm{GPa})\end{array}$ & $\begin{array}{c}\mathrm{MOE} \\
(\mathrm{GPa})\end{array}$ \\
Media muestral & 8,62 & 7,04 \\
Error estándar & 0,282 & 0,237 \\
Asimetría & 0,372 & 0,319 \\
Curtosis & $-1,550$ & $-1,252$ \\
Tamaño de la muestra & 18 & 18
\end{tabular}


presenta en la tabla 1, es inferior a los datos bibliográficos de especies latifoliadas. Debido a que no se encontró información acerca del Módulo de Elasticidad en vibración transversal para maderas de Prosopis sp., el valor de Ed no puede ser comparado con datos del mismo género.

El valor de la media muestral para el Módulo de Elasticidad estático, presentado en la tabla 1, se sitúa por abajo del intervalo de valores de MOE, para especies de madera del mismo género encontrados en la bibliografía. De acuerdo con Bodig y Goodman (1973), y Guitard y El Amri (1987), la resistencia mecánica de la madera es función creciente de su densidad. El valor promedio de la densidad de la madera de Prosopis calculado de $730\left(\mathrm{~kg} / \mathrm{m}^{3}\right)$, es también inferior a los valores de densidad encontrados para este género en la bibliografía. Lo que implica que los valores de MOE calculados son inferiores pero proporcionales a los reportados en la bibliografía.

\section{Comportamiento viscoelástico}

En un primer enfoque de análisis por grupos, según el tipo de ensayo, los resultados de la tabla 1 muestran que los valores del Módulo de Elasticidad dinámico son superiores al valor correspondiente del Modulo de Elasticidad estático. Por otra parte, si se analizan los resultados particulares para cada probeta presentados en la Figura 5, se distingue que el valor de Ed calculado en cada una de ellas es mayor que el respectivo valor calculado de MOE en la misma probeta, esta diferencia entre valores fue observada en cada una de las probetas examinadas, el cálculo de la diferencia entre los valores es de $22 \%$ en promedio. Es importante mencionar que en la Figura 5, los valores respectivos para cada especimen son correspondientes, es decir, que siguen el mismo patrón y son propor- cionales entre sí. El valor de Ed es mayor que el valor de MOE, para cada una de las probetas estudiadas, donde el único parámetro experimental que varió fue la velocidad de solicitación.

La prueba de comparación de medias entre los valores del Módulo de Elasticidad dinámico y estático, resultó en un intervalo de confianza estimado entre 0,8297 y 2,3236 . Además, el valor de $p$ calculado $(0,00014)$ por la prueba $t$ de Student para muestras independientes, es menor que 0,05 , lo cual permite rechazar la hipótesis nula $\left(\mathrm{H}_{0}\right.$ : $\left.\grave{i}_{1}=\grave{i}_{2}\right)$. Para los grupos dinámico y estático, estos resultados representan una diferencia estadística significativa entre los dos grupos de ensayos para un nivel de confiabilidad del $95 \%$, explicando de esta manera la diferencia de los valores probeta a probeta, pero no entre muestras pares.

De acuerdo con Bodig y Jayne (1993), la diferencia entre los resultados es explicada por el carácter viscoelástico de la madera, que aumenta la rigidez aparente de las probetas debido a que la velocidad de carga en los métodos dinámicos es superior a la velocidad de carga en el método estático. Las propiedades viscoelásticas de la madera y la resistencia mecánica aparente en el ensayo de vibración transversal es la superposición de la respuesta de su rigidez elástica más la respuesta de la rigidez viscosa, que se observa únicamente en ensayos donde la velocidad de solicitación es superior a la velocidad de la solicitación de un ensayo estático equivalente. El valor promedio de las frecuencias ( $f$ ) medidas en los ensayos dinámicos utilizando probetas normalizadas, fue en promedio de $682 \mathrm{~Hz}$, mientras que la velocidad de carga promedio de $1 \mathrm{~mm} / \mathrm{min}$ fue la empleada para los ensayos estáticos. 


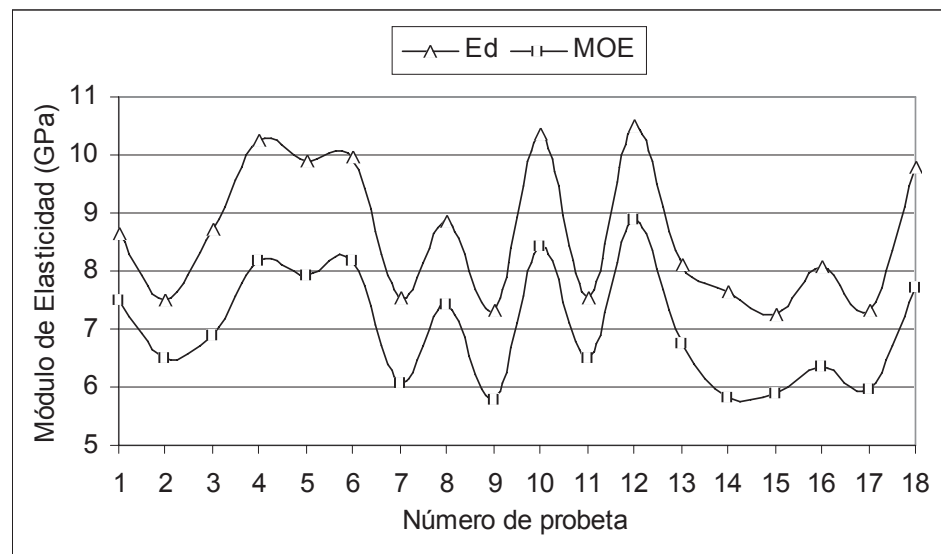

Figura 5. Gráfico de los valores de los Módulos de Elasticidad dinámico y estático.

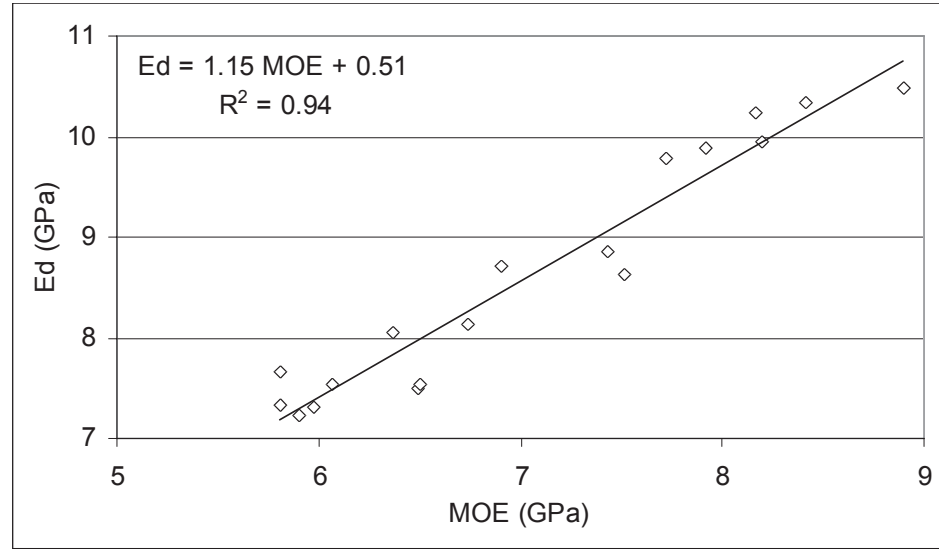

Figura 6. Diagrama de dispersión entre los Módulos de Elasticidad estático y dinámico.

El análisis del diagrama de dispersión entre los valores de MOE y Ed presentados en la Figura 6, resulta en un coeficiente de correlación $\mathrm{R}^{2}$ cercano a la unidad, resultado que confirma una correspondencia lineal de los ensayos. Este resultado es comparable al publicado anteriormente por los autores citados en la introducción, y permite confirmar la robustez de la metodología aplicada y su utilidad en investigaciones del mismo género.
De la observación de la Figura 6, se desprende que el MOE puede ser un buen predictor de Ed y viceversa. Además, el valor del coeficiente de correlación de Pearson $r$ de 0.97 , permite afirmar que si aumenta el valor del MOE de la madera de Prosopis sp., los valores de Ed se incrementan proporcionalmente, resultados que concuerdan con las conclusiones publicadas con anterioridad por Görlacher (1984) y Machek y et al. (2001), entre otros investigadores. 
La aplicación práctica de este resultado es la utilización del Módulo de Elasticidad dinámico como predictor para estimar el Módulo de Elasticidad estático, que es al mismo tiempo un parámetro referente para evaluación, de acuerdo con Bodig (1994) y Beall (1999). Además, el Módulo de Elasticidad dinámico es útil en la concepción de estructuras de madera donde las cargas dinámicas, tales como vibraciones y sismos, son críticas.

\section{CONCLUSIONES}

La estrategia experimental propuesta en esta investigación tuvo por objeto realizar ensayos exploratorios no destructivos y de manera intensiva sobre una muestra de madera de Prosopis sp., lo que permitió obtener características similares de resistencia mecánica entre ensayos equivalentes, donde sólo varió la velocidad de carga. Los ensayos dinámicos practicados resultaron ser confiables, son más rápidos y sus parámetros medidos son más repetitivos en comparación con los ensayos estáticos.

La investigación fue un estudio de caso y una extensión de sus resultados es la aplicación de la metodología analizando otras especies. Además se recomienda utilizar ensayos de carácter no destructivo con probetas de diferentes dimensiones o ensayando elementos de madera con dimensiones de uso.

\section{REFERENCIAS}

American Institute of Timber Construction. 1994. Timber construction manual. $4^{a}$ Ed. John Wiley \& Sons, Inc. EUA. $904 \mathrm{p}$.

Ashby, M.F. 1999. Materials selection in mechanical design. $2^{\mathrm{a}}$ Ed. Butter-
worth-Heinemann, Gran Bretaña. 502 p.

Beall, F.C. 1999. Future of nondestructive evaluation of wood and wood-based materials. The e-Journal of Nondestructive Testing \& Ultrasonics 4 (11). 9 p. Disponible en: http://www. ndt.net.

Bodig, J. 1994. NDE of wood in North America - Concepts and applications. In: First European Symposium on Nondestructive Evaluation of Wood. University of Forestry and Wood Science. Hungría. 15 p.

Bodig, J. y J.R. Goodman. 1973. Prediction of elastic parameters for wood. Wood Science (5)4:249-264.

Bodig, J. y B.A. Jayne. 1993. Mechanics of wood composites. Kreiger Publishing Company, EUA. 712 p.

Forest Products Laboratory. 1999. Wood handbook. Wood as an engineering material. Gen. Tech. Rep. FPL-GTR113. Department of Agriculture. Forest Service. Forest Products Laboratory. EUA. 463 p.

Goens, E. 1931. Determination of Young's modulus from flexural vibrations. Annalen der Physik 11(6):649-678.

Görlacher, R. 1984. Ein neues Messverfahren zur Bestimmung des Emodulus von Holz. Holz als Roh-und Werkstoff (42):212-222.

Guitard, D. 1987. Mécanique du matériau mois et composites. CEPADUESEDITIONS, Francia. 238 p.

Guitard, D. y F. El Amri. 1987. Modèles prévisionnels de comportement élastique tridimensionnel pour les bois feuillus et les bois résineux. Annales des Sciences Forestières 44(3):335-358. 
Haines, D.W., J.M. Leban y C. Herbé. 1996. Determination of Young`s modulus for spruce, fir and isotropic materials by the resonance flexure method with comparisons to static flexure and other dynamic methods. Wood Science and Technology (30):253-263.

Hearmon, R.F.S. 1966. Theory of the vibration testing of wood. Forest Products Journal 16(8):29-40.

Ilic, J. 2001. Relations among the dynamic and static elastic properties of air-dry Eucalyptus delegatensis R. Baker. Holz als Roh- und Werkstoff (59):169-175.

International Organization for Standardization ISO 3129-1975 (E). 1975a. Wood sampling methods and general requirements for physical and mechanical tests. Iso Catalog 79 Wood technology; 79.040 Wood, sawlogs and saw timber. International Organization for Standardization (ISO). Disponible en: www.iso.ch/iso/en/IsoOnline.openerpage

International Organization for Standardization ISO 3349-1975 (E). 1975b. Wood - Determination of modulus of elasticity in static bending. ISO Catalog 79 Wood technology; 79.040 Wood, sawlogs and saw timber. International Organization for Standardization (ISO). Disponible en: www.iso.ch/iso/en/ISOOnline. openerpage.

Jayne, B.A. 1959. Vibrational properties of wood as indices of quality. Forest Products Journal 9(11):413-416.

Kaiserlik, J.H. y R.F. Pellerin. 1977. Stress wave attenuation as an indicator of lumber strength. Forest Products Journal 27(6):39-43.
Kollmann, F. y H. Krech. 1960. Dynamische Messung der elastischen Holzeigenschaften und der Dämpfung. Holz Roh-Werkstoff (18):41-54.

Kollmann, F.P. y W.A. Côté. 1968. Principles of wood science and technology. Volume I: Solid wood. SpringerVerlag, Alemania. 592 p.

Lemmens, J.W. (s/f). Operating instructions for the GrindoSonic MK5 "Industrial" Instrument. J.W. Lemmens, Inc. EUA. 25 p.

Machek, L., H. Militz y R. Sierra-Alvarez. 2001. The use of an acoustic technique to assess wood decay in laboratory soil-bed tests. Wood Science and Technology 34(6):467-472.

Panshin, A.J. y C. De Zeeuw. 1964. Textbook of wood technology. Volume I. McGraw-Hill, Inc. EUA. 705 p.

Pellerin, R.F. 1965. A vibrational approach to nondestructive testing of structural lumber. Forest Products Journal 15(3):93-101.

Perstorper, M. 1992. Predicting the stiffness of structural timber using dynamic modal tests. In: Proceedings of IUFRO S5.02 Timber Engineering 1992 Conference. Francia. pp: 305-324.

Ross, R.J. et al. 1977. Relationship between log and lumber modulus of elasticity. Forest Products Journal 47(2):89-92.

Ross, R.J. y R.F. Pellerin. 1988. NDE of wood-based composites with longitudinal stress waves. Forest Products Journal 38(5):39-45.

Ross, R.J. y R.F. Pellerin. 1994. Nondestructive testing for assessing wood members in structures: A review. 
Gen. Tech. Rep. FPL-GTR-70 (Rev.). Department of Agriculture. Forest Service. Forest Products Laboratory. EUA. $40 \mathrm{p}$.

Sandoz, J.L. 2000. Wood testing using Acousto-Ultrasonic. Publication IBOIS 00:23, Institut de Statique et Structures IBOIS. Construction en bois. In: Proceedings of the World Conference on Timber Engineering (WCTE 2000), Whistler Resort, British Columbia, Canada. 6 p.
Sandoz, J.L. 2002. High performance timber by ultrasonic grading. Publication IBOIS 00:20, Institut de Statique et Structures IBOIS. Construction en Bois. In: Proceedings of the $7^{\text {th }}$ World Conference on Timber Engineering (WCTE 2002). MARA University of Technology, Selangor, Malasia. $7 \mathrm{p}$.

Timoshenko, S., D.H. Young y W. Weaver. 1994. Vibration problems in engineering. John Wiley, Nueva York. 
